\title{
Vibrational Relaxation and Hydrogen-Bond Dynamics of $\mathrm{HDO}: \mathrm{H}_{2} \mathrm{O}$
}

\author{
Michel F. Kropman,* Han-Kwang Nienhuys, Sander Woutersen, and Huib J. Bakker \\ FOM Institute for Atomic and Molecular Physics, Kruislaan 407, 1098 SJ Amsterdam, The Netherlands
}

Received: January 5, 2001

\begin{abstract}
Femtosecond two-color mid-infrared pump-probe spectroscopy is used to study the vibrational relaxation and the hydrogen-bond dynamics of $\mathrm{HDO}$ dissolved in liquid $\mathrm{H}_{2} \mathrm{O}$. By looking at the spectral dynamics of the OD stretch mode, direct information on the hydrogen-bond dynamics of the $\mathrm{H}_{2} \mathrm{O}$ solvent is obtained. By fitting the data using the Brownian oscillator model, we determined the vibrational lifetime of the OD stretch vibration and the hydrogen-bond length correlation time. The hydrogen-bond correlation time of $\mathrm{H}_{2} \mathrm{O}$ is significantly shorter than for $\mathrm{D}_{2} \mathrm{O}$, found previously.
\end{abstract}

\section{Introduction}

Infrared and Raman spectroscopy have long been used to study liquid water. ${ }^{1,2}$ Unfortunately, these conventional linear spectroscopic techniques do not give information on the dynamics of the water molecules because, due to the large variation in hydrogen-bond lengths, the vibrational absorption bands are strongly inhomogeneously broadened. Advancements in laser technology have enabled the use of nonlinear spectroscopic techniques. However, to probe dynamical behavior with these techniques, the pulse duration has to be shorter than the time scale of the phenomenon under consideration.

The vibrational modes in water are optically active in the mid-infrared. ${ }^{3}$ At these wavelengths, no laser sources are available that deliver short and powerful pulses. Using frequencyconversion processes ${ }^{4}$ in nonlinear crystals such as $\mathrm{LiNbO}_{3}$, the available wavelengths (e.g., $1064 \mathrm{~nm}$ for $\mathrm{Nd}$ :YAG lasers or 800 $\mathrm{nm}$ for Ti:sapphire lasers) can be converted into mid-infrared light. This conversion requires a high-intensity source laser; the experiments that can be performed are thus limited by the pulse duration and energy of the pulses of the source laser.

In the 1970s, Nd:glass ${ }^{5}$ and $\mathrm{Nd}: \mathrm{YAG}^{6}$ lasers were developed, producing powerful picosecond pulses, typically $1 \mathrm{~mJ}$ and $6 \mathrm{ps}$ for Nd:glass, and $40 \mathrm{~mJ}$ and $20 \mathrm{ps}$ for Nd:YAG. The lasers had a fairly low repetition rate, $10 \mathrm{~Hz}$ for $\mathrm{Nd}$ :YAG and only $1 \mathrm{~Hz}$ for Nd:glass, which made it very difficult to obtain data within an acceptable signal-to-noise ratio. Nevertheless, with these lasers it became possible to study the dynamics of vibrational excitations in monomers such as hydrogen chloride $(\mathrm{HCl})^{6}$ and chloroform $\left(\mathrm{CHCl}_{3}\right){ }^{7}$ and ethanol clusters $\left(\mathrm{C}_{2} \mathrm{H}_{5} \mathrm{OH}\right)^{8}$ dissolved in carbon tetrachloride $\left(\mathrm{CCl}_{4}\right)$. With the advent of Ti:sapphire lasers, that produce powerful subpicosecond laser pulses at a 1 $\mathrm{kHz}$ repetition rate, the study of faster processes occurring in hydrogen-bonded species such as liquid water ${ }^{9-14}$ and ethanol ${ }^{15}$ came within reach.

The study of water is complicated by the hydrogen-bond structure, and by the strong coupling between $\mathrm{OH}$ groups of neighboring molecules. ${ }^{13,14}$ Furthermore, the symmetric and antisymmetric stretching vibrations in liquid $\mathrm{H}_{2} \mathrm{O}$ are spectroscopically nearly indistinguishable. To avoid these complications, experimental work has mainly been devoted to dilute

* To whom correspondence should be addressed. E-mail: m.kropman@ amolf.nl. solutions of $\mathrm{HDO}$ in $\mathrm{D}_{2} \mathrm{O}, 1,9,11,12,16$ where the $\mathrm{OH}$ and $\mathrm{OD}$ stretching bands are well separated, being centered at 3400 and $2500 \mathrm{~cm}^{-1}$, respectively, while the chemical (hydrogen-bonded) structure remains nearly unchanged.

Using femtosecond mid-infrared pump-probe spectroscopy, it was shown that the $\mathrm{OH}$ stretching vibration of $\mathrm{HDO}$ in $\mathrm{D}_{2} \mathrm{O}$ has a lifetime of approximately $740 \mathrm{fs} .{ }^{12,14}$ In other studies, the hydrogen-bond dynamics was studied by looking at the timedependent signal at frequencies different from the excitation frequency. ${ }^{11,16}$ It was found that the $\mathrm{OH}$ stretching frequency, and therefore the hydrogen-bond length, is not constant in time, but rather changes on a time-scale of approximately $500 \mathrm{fs}$. These hydrogen-bond dynamics, however, are determined by the $\mathrm{D}_{2} \mathrm{O}$ "solvent". To study the hydrogen-bond dynamics of $\mathrm{H}_{2} \mathrm{O}$ ("real water"), we would like to probe the $\mathrm{OH}$ stretching vibration in pure $\mathrm{H}_{2} \mathrm{O}$. Unfortunately, as a result of rapid intermolecular energy transfer, ${ }^{13,14}$ the $\mathrm{OH}$ stretching vibration is not localized on one $\mathrm{OH}$ oscillator for a sufficient period of time. Therefore, it is not possible to obtain information on the hydrogen-bond dynamics of liquid water by probing the $\mathrm{OH}$ stretch in pure $\mathrm{H}_{2} \mathrm{O}$. However, by looking at the OD stretching vibration of $\mathrm{HDO}$ in $\mathrm{H}_{2} \mathrm{O}$, information on these dynamics can be obtained.

\section{Experiment}

To perform time-resolved two-color pump-probe spectroscopy on the OD stretching vibration, we need powerful femtosecond pump and probe pulses at wavelengths near 4000 $\mathrm{nm}$. The pump pulses are generated using the setup shown in Figure 1. A commercial Ti:sapphire regenerative/multipass amplifier delivers pulses of $800 \mathrm{~nm}$ wavelength, $3 \mathrm{~mJ}$ energy, $100 \mathrm{fs}$ duration, at a $1 \mathrm{kHz}$ repetition rate. Part of this $800 \mathrm{~nm}$ light $(0.9 \mathrm{~mJ})$ is used to pump an optical parametric generation and amplification stage (OPA) based on $\beta$-barium borate (BBO), that can be tuned from 1.1 to $2.6 \mu \mathrm{m}$. In our experiment, it is tuned to approximately $1330 \mathrm{~nm}$ (signal) and $2000 \mathrm{~nm}$ (idler). The idler is subsequently frequency-doubled to $1000 \mathrm{~nm}$ using a second BBO crystal. The $1000 \mathrm{~nm}$ light is used as seed in a second parametric amplification process, where it is combined with a fresh part $(1.4 \mathrm{~mJ})$ of the $800 \mathrm{~nm}$ light in a $5 \mathrm{~mm}$ potassium niobate $\left(\mathrm{KNbO}_{3}\right)$ crystal to produce $4000 \mathrm{~nm}(2500$ $\mathrm{cm}^{-1}$ ) pulses. Residual seed and pump light is filtered out of the beam using dielectric $800 \mathrm{~nm}$ and YAG mirrors, and a long 


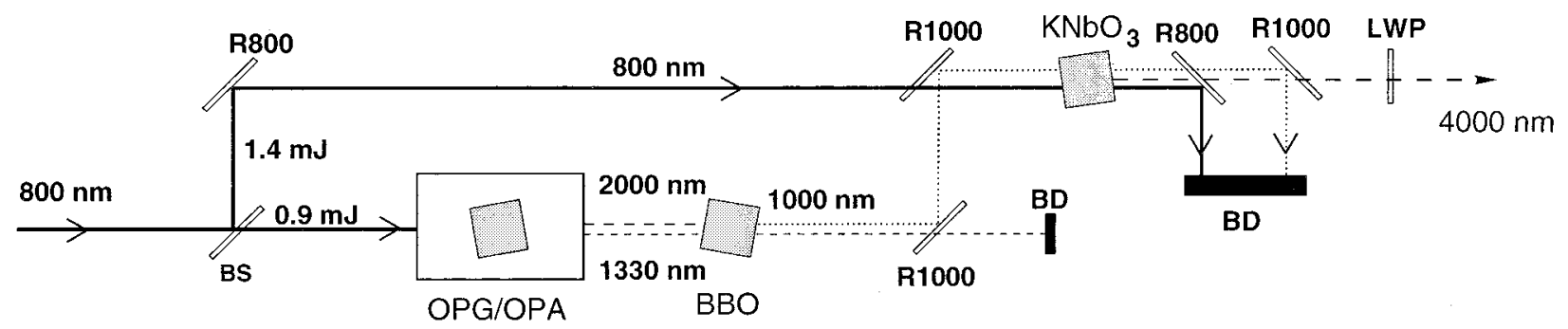

Figure 1. Generation of $4 \mu \mathrm{m}$ pump pulses. Abbreviations: $\mathrm{BBO}, \mathrm{KNbO}_{3}$ : crystals; $\mathrm{R} 800, \mathrm{R} 1000$ : dielectric $800 \mathrm{~nm}$, broadband $900-1100 \mathrm{~nm}$ mirrors; LWP: long wave pass-filter, BD: beam dump, OPG/OPA: 3-pass optical parametric generation and amplification stage based on BBO.

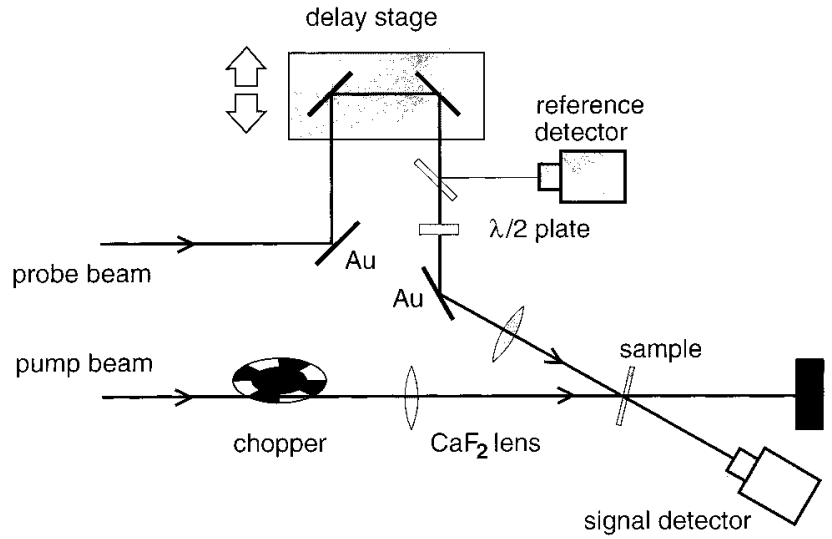

Figure 2. Pump-probe setup. The signal detector measures the total intensity of the delayed probe pulse, divided by the intensity measured by the refererence detector, to account for the pulse-to-pulse intensity variations. The chopper blocks every other pump pulse, to obtain the pump-induced absorption change of the sample. Gold mirrors and $\mathrm{CaF}_{2}$ plates and lenses are used to direct and focus the beams. A $\lambda / 2$ plate rotates the probe beam polarization to exclude effects of reorientation.

wave pass filter. By tuning the seed pulses, the output pulses are tuned from 3700 to $4250 \mathrm{~nm}\left(2350-2700 \mathrm{~cm}^{-1}\right)$, with a typical energy of $18 \mu \mathrm{J}$, and a bandwidth of $70 \mathrm{~nm}$.

The probe pulses at wavelengths below $4250 \mathrm{~nm}$ are generated nearly the same way. A second OPA based on a BBO crystal is pumped with $0.4 \mathrm{~mJ} 800 \mathrm{~nm}$ pulses. Its output at 2000 $\mathrm{nm}$ is converted into $1000 \mathrm{~nm}$ using a second BBO crystal. For the mid-infrared generation, we now use $\mathrm{LiNbO}_{3}$, since with this crystal the spectral shape and pulse energy of the generated light are more stable than with a $\mathrm{KNbO}_{3}$ crystal. It is pumped with $0.1 \mathrm{~mJ} 800 \mathrm{~nm}$ light. Cross-correlation traces of these pulses with the pump pulses (delay-dependence of sumfrequency generation in a $\mathrm{LiIO}_{3}$ crystal), have a fwhm of 350 fs (pulse duration of $250 \mathrm{fs}$ ).

Because of the absorbance in $\mathrm{LiNbO}_{3}$ at longer wavelengths, we use a different method to generate (probe) wavelengths longer than $4250 \mathrm{~nm}$. The OPA is now pumped with $0.6 \mathrm{~mJ}$ of $800 \mathrm{~nm}$ light. For the generation of the $4400 \mathrm{~nm}$ pulses used in the present experiment, it is tuned to 1350 (signal) and 1950 $\mathrm{nm}$ (corresponding idler). In a $3 \mathrm{~mm} \mathrm{AgGaS}_{2}$ crystal, signal and idler are used in a difference-frequency generation process. Cross-correlation traces of these pulses have a fwhm of $600 \mathrm{fs}$.

The pump and probe pulses are used in a pump-probe setup, shown in Figure 2. The pump excites a significant fraction of the molecules, causing an absorption change that is measured by the probe beam. The absorption will decrease when the probe is tuned to some frequency within the $0 \rightarrow 1$ (the numbers refer to vibrational levels) absorption band, and increase for frequencies in the $1 \rightarrow 2$ band (due to the anharmonicity of the OD stretching potential function, the $1 \rightarrow 2$ transition is red-shifted by approximately $200 \mathrm{~cm}^{-1}$ with respect to the $0 \rightarrow 1$ transition).

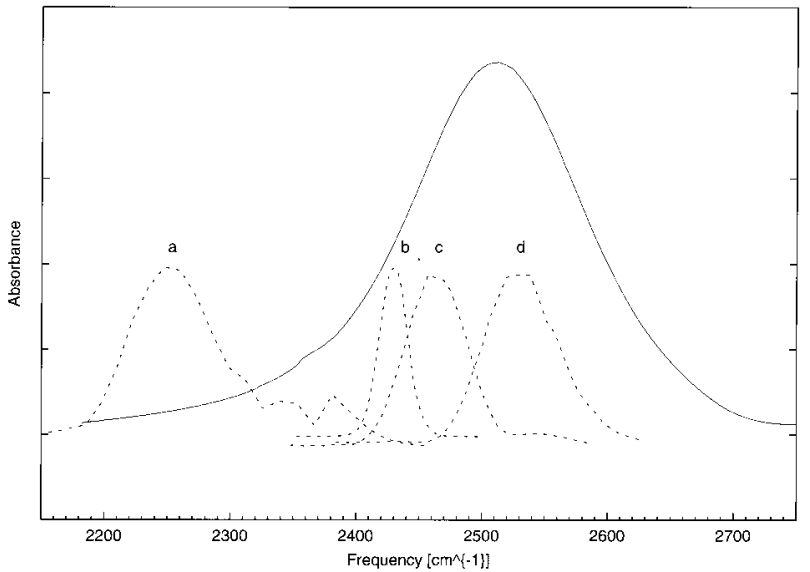

Figure 3. Absorption band of the OD stretching vibration in HDO: $\mathrm{H}_{2} \mathrm{O}$, corrected for the $\mathrm{H}_{2} \mathrm{O}$ background (solid line). Also shown are the power spectra of the probe pulses used in the experiments (dotted lines; the letters correspond to the ones in Figure 4).

Part of the probe beam is split off using a $\mathrm{CaF}_{2}$ plate and measured by a reference detector, to account for the pulse-topulse variations in probe energy. The pump and probe beams are focused in the sample using $+175 \mathrm{~mm}$ and $+100 \mathrm{~mm} \mathrm{CaF}_{2}$ lenses, respectively; the focal lengths differ to ensure that the probe beam focus is smaller than the focus of the pump. The intensities of the transmitted probe pulses and of the reference pulses are measured by PbSe detectors. A chopper is placed in the pump beam, blocking every other pump pulse. In this way, the transmitted probe pulse intensity can be measured with $(I)$ or without the pump pulse $\left(I_{0}\right)$. The transmission $T$ of the probe beam with (without) pump beam is then given by $I / I_{\text {ref }}\left(I_{0} / I_{\text {ref }}\right)$. The absorption change, $\Delta \alpha=-\ln \left(T / T_{0}\right)$ can now be measured as a function of the time delay between pump and probe. The polarization of the probe beam, originally parallel to the pump beam, is rotated over the magic angle $\left(54.7^{\circ}\right)$ to exclude effects of reorientation.

The sample used was a $50 \mu \mathrm{m}$ thick layer of about $5 \%$ HDO in $\mathrm{H}_{2} \mathrm{O}$. Sample thickness and concentration were chosen such that the transmission at the center of the OD absorption band was approximately 5\%. During the measurements, the sample was continuously rotated to avoid heating effects due to previous pulses.

\section{Results}

The absorption band of the OD stretching vibration is centered at $2500 \mathrm{~cm}^{-1}$ and has a fwhm of $170 \mathrm{~cm}^{-1}$ (see Figure 3). To investigate the relaxation of the OD stretching vibration, we recorded delay scans at different pump and probe frequencies. They are shown in Figure 4, where the absorption change (ln$\left.\left(T / T_{0}\right)\right)$ is plotted as a function of the time delay between the pump and probe pulses, for several pump and probe frequencies. It is observed that the pump-probe signal strongly depends on the employed frequencies. 

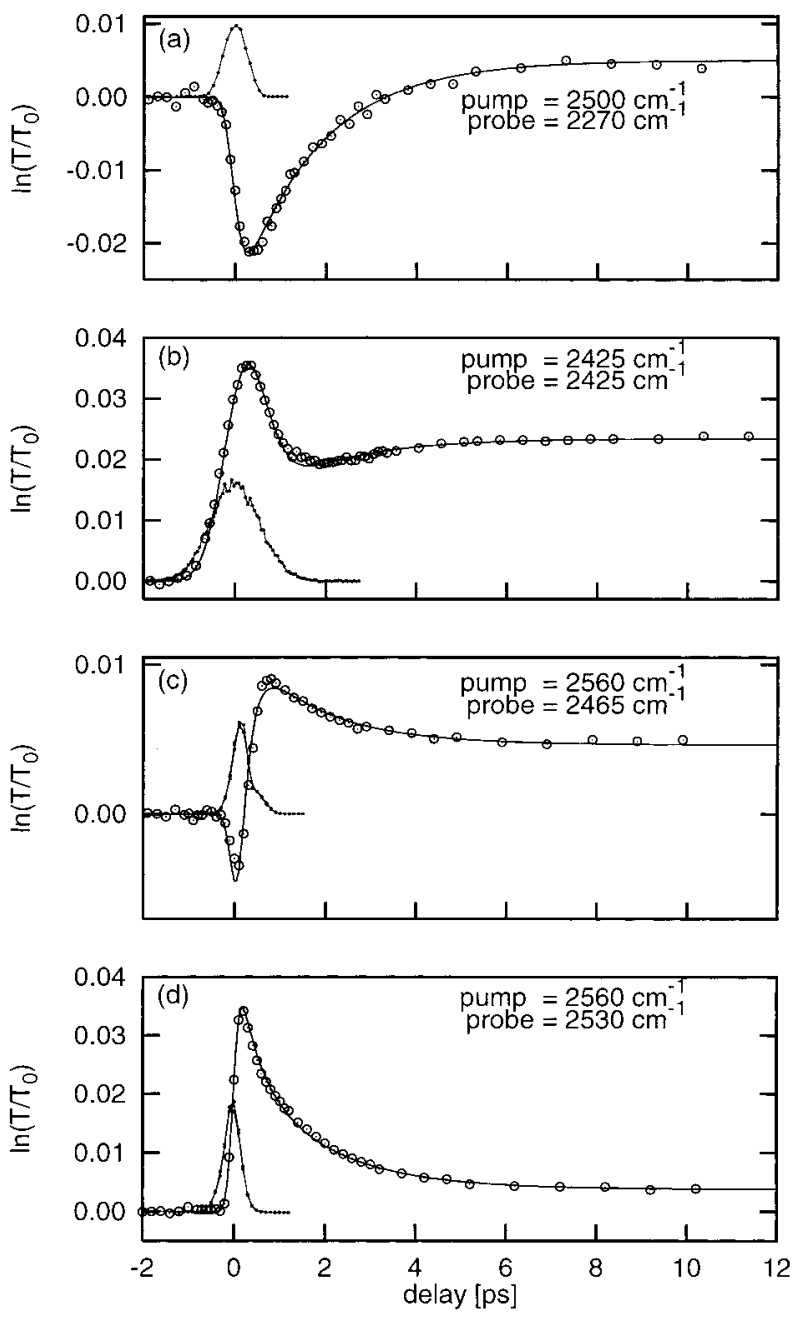

Figure 4. Measured pump-probe transients of $\mathrm{HDO}: \mathrm{H}_{2} \mathrm{O}$ at different pump and probe frequencies. The solid line is a fit to the data (see Section 4). Also shown are cross-correlation traces of the pump and probe pulses.

At delays shorter than $1 \mathrm{ps}$, very complex behavior is observed: in Figures $4 \mathrm{~b}$ and $4 \mathrm{~d}$, a bleaching signal is observed; Figure $4 \mathrm{a}$ shows an induced absorption due to the $1 \rightarrow 2$ transition. In Figure 4c, within $1 \mathrm{ps}$, the initial absorption changes to a bleaching signal. After $1 \mathrm{ps}$, all signals either increase (Figures $4 \mathrm{a}$ and $4 \mathrm{~b}$ ) or decrease (Figures $4 \mathrm{c}$ and $4 \mathrm{~d}$ ) exponentially to a decreased absorption level at the same time scale of $\sim 2$ ps.

The persistent absorption change at large delays is due to the temperature increase that results from the thermalization of the pump pulse energy. Raising the temperature causes the absorption band to shift to the blue and become weaker. ${ }^{17}$ This gives rise to an absorption decrease at most frequencies, except at high frequencies, where a final absorption increase is observed (not shown).

\section{Model}

The short-time behavior, within $1 \mathrm{ps}$, depends strongly on both pump and probe frequencies. For instance, the initial absorption at a $2465 \mathrm{~cm}^{-1}$ probe frequency (Figure 4c) is not observed when pumping at 2465 or $2500 \mathrm{~cm}^{-1}$, instead of 2560 $\mathrm{cm}^{-1}$. The bleaching at $2425 \mathrm{~cm}^{-1}$ (Figure $4 \mathrm{~b}$ ) is not observed when pumping at $2560 \mathrm{~cm}^{-1}$; with this combination of pump and probe frequencies, an induced absorption signal is observed. Because of the dependence of the fast signal on both pump and

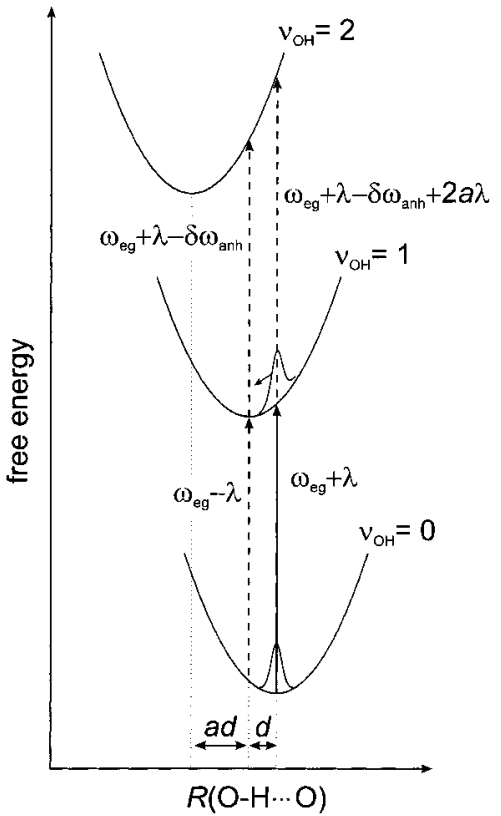

Figure 5. Harmonic potential energy functions for the OD stretching vibration (ground state and the first two excited states), as a function of the $\mathrm{O}-\mathrm{H} \cdots \mathrm{O}$-distance (see Section 4). $\omega_{\mathrm{eg}}+\lambda$ is the ground-state absorption center frequency, $2 \lambda$ the Stokes shift, $\delta \omega_{\text {anh }}$ the anharmonic frequency shift; $d$ is the displacement of the potential minimum between $v_{\mathrm{OH}}=0$ and $\nu_{\mathrm{OH}}=1 ; a d$ is the displacement of the minima of $v_{\mathrm{OH}}=$ 1 and $v_{\mathrm{OH}}=2$.

probe frequencies, we assign it to a spectral diffusion process, as will be explained below. In contrast, the sign of the slow component only depends on the probe, and not on the pump frequency. This means that for the slow component (i.e., after $1 \mathrm{ps}$ ), the spectral diffusion has completed, so that the slow component corresponds to the actual lifetime of the vibration.

In our experiment, the pump pulse selectively excites the OD stretching vibration of a subset of the HDO molecules. The subset is characterized by a small range of OD stretching frequencies (corresponding to a particular range of hydrogenbond lengths ${ }^{18}$ ). The $v=1$ state is thus populated, and the ground-state depleted, which will change the absorbance at several frequencies. Due to the random motion of the solvent water molecules, the spectral shapes of both ground-state depletion ("holes") and excited-state population ("particles") will broaden and shift toward their respective equilibrium band shapes (spectral diffusion). Excited molecules will after some time (lifetime $T_{1}$ ) relax to the ground state, thereby locally raising the temperature, which results in a weakening and blueshift of the absorption band.

All the above features are incorporated in the Brownian oscillator model, that describes the time-dependent two-color pump-probe signal of a high-frequency molecular vibration coupled to a strongly damped, harmonic, low-frequency hydrogenbond mode. For an excited $\mathrm{OH}$ stretching vibration, the hydrogen-bond potential is shifted over a distance $d$ to shorter hydrogen-bond lengths compared to the potential in the vibrational ground state (see Figure 5). Thus, when the $\mathrm{OH}$ stretch mode is excited, the hydrogen bond will contract, and the $\mathrm{OH}$ stretch frequency will show a dynamical Stokes shift to lower frequencies. The relation between the absorption band shape $e^{-\omega^{2} / 2 \Delta^{2}}$ and the Stokes shift $2 \lambda$ is given by ${ }^{19}$

$$
2 \lambda=\hbar \Delta^{2} / k_{\mathrm{B}} T
$$

The hydrogen-bond mode in liquid water is strongly over- 
damped. Therefore, its motion is diffusive, and can be described by a correlation function (in terms of the oscillation frequency) of the form

$$
\langle\delta \omega(\tau) \delta \omega(0)\rangle=\Delta^{2} e^{-\tau / \tau_{\mathrm{c}}}
$$

in which $\tau_{\mathrm{c}}$ is the spectral diffusion correlation time, and $\Delta$ the root-mean-squared amplitude of the frequency fluctuations.

For the pump-probe signal, a formula can be derived giving the time-dependent absorption change as a function of pump and probe frequency. ${ }^{19,20} \mathrm{We}$ have added a term to account for the excited-state absorption and an overall factor of $\exp (-\tau /$ $T_{1}$ ) for the finite lifetime of the excitation. Another term was added to describe the effect of temperature increase on the pump-probe signal; it was simply taken proportional to the fraction of the molecules that have decayed from the $v=1$ state, after which the vibrational energy has become thermal. The first three terms describe the particle, hole, and induced absorption contributions, respectively. The fourth term represents the temperature-induced absorption change.

$$
S_{\mathrm{PP}}\left(\omega_{1}, \omega_{2}, \tau\right)=F(\tau)\left\{S_{\mathrm{p}}(\tau)+S_{\mathrm{h}}(\tau)+S_{\mathrm{ea}}(\tau)\right\}+S_{T}(\tau)
$$

where

$$
\begin{gathered}
F(\tau)=\frac{2 \pi e^{-\tau / T_{1}}}{\sqrt{\left(\Delta^{2}+w_{1}^{2}\right) \alpha^{2}(\tau)}} e^{-\left(\omega_{1}-\omega_{\mathrm{eg}}^{0}-\lambda\right)^{2} / 2\left(\Delta^{2}+w_{1}^{2}\right)} \\
S_{\mathrm{p}}(\tau)=e^{-\left(\omega_{2}-\omega_{e}(\tau)\right)^{2} / 2 \alpha^{2}(\tau)} \\
S_{\mathrm{h}}(\tau)=e^{-\left(\omega_{2}-\omega_{\mathrm{g}}(\tau)\right)^{2} / 2 \alpha^{2}(\tau)} \\
S_{\mathrm{ea}}(\tau)=\sigma_{\mathrm{ea}} e^{-\left(\omega_{2}-\omega_{\mathrm{ea}}(\tau)\right)^{2} / 2 a^{2} \alpha^{2}(\tau)} \\
S_{T}(\tau)=\Delta \sigma\left(\omega_{2}\right)\left(1-e^{-\tau / T_{1}}\right) \\
\omega_{\mathrm{e}}(\tau)=\omega_{\mathrm{eg}}^{0}-\lambda+e^{-\tau / \tau_{\mathrm{c}}}\left(\omega_{0}-\omega_{\mathrm{eg}}^{0}+\lambda\right) \\
\omega_{\mathrm{g}}(\tau)=\omega_{\mathrm{eg}}^{0}+\lambda+e^{-\tau / \tau_{\mathrm{c}}}\left(\omega_{0}-\omega_{\mathrm{eg}}^{0}-\lambda\right) \\
\omega_{\mathrm{ea}}(\tau)=\omega_{\mathrm{eg}}^{0}+\lambda-\delta \omega_{\mathrm{anh}}+a e^{-\tau / \tau_{\mathrm{c}}}\left(\omega_{0}-\omega_{\mathrm{eg}}^{0}+\lambda\right) \\
\alpha^{2}(\tau)=\Delta^{2}\left[1-\frac{\Delta^{2}}{\Delta^{2}+w_{1}^{2}} e^{-2 \tau / \tau_{\mathrm{c}}}\right]+w_{2}^{2} \\
\omega_{0}=\omega_{1} \frac{\Delta^{2}}{\Delta^{2}+\omega_{1}^{2}}+\left(\omega_{\mathrm{eg}}^{0}+\lambda\right) \frac{w_{1}^{2}}{\Delta^{2}+w_{1}^{2}} \\
\end{gathered}
$$

$\omega_{1}, w_{1}$ and $\omega_{2}, w_{2}$ are the central frequencies and the spectral widths of the Gaussian pump and probe pulses, respectively. $\omega_{\text {eg }}+\lambda$ is the center frequency of the absorption band. $\omega_{\mathrm{e}}$ and $\omega_{\mathrm{g}}$ denote the time-dependent center frequencies of the particle and hole distributions.

All data can be described by eq 3 , convolved with the crosscorrelation trace, using a $T_{1}$ of $1.8 \mathrm{ps}$, a spectral diffusion correlation time of $400 \mathrm{fs}$, central frequencies of 2520 and 2350 $\mathrm{cm}^{-1}$, and widths of $170 \mathrm{~cm}^{-1}$ and $240 \mathrm{~cm}^{-1}$ for the $0 \rightarrow 1$ and $1 \rightarrow 2$ transitions, respectively. The cross-section ratio $\sigma_{\text {ea }}$ of the excited-state absorption relative to the ground-state absorption was found to be 1.38 .
The fit results are shown in Figure 4 as solid lines. It is seen that all results are well described, including Figures $4 \mathrm{~b}$ and $4 \mathrm{c}$, that are rich in features. Pumping at the red side of the absorption band, $2425 \mathrm{~cm}^{-1}$ (Figure 4b), leads to a population in the $v=$ 1 state, and a depletion of the ground state, that are both centered at the short-hydrogen-bond side of their respective minima (see Figure 5). Both populations will evolve in time, predominantly toward their potential minima. Directly after the pump, before these populations have shifted, the probe will see a bleaching signal due to the ground-state depletion. The time evolution of the particle and hole distributions is such that the $0 \rightarrow 1$ bleaching will shift away from the probe frequency, while the $1 \rightarrow 2$ absorption moves toward the probe frequency. Both processes result in a decrease of the bleaching signal. As the excitation itself will decay, giving rise to a temperature increase, the absorption change will reach its new equilibrium value. Between the last two counteracting processes, a minimum in the absorption change is reached.

In Figure 4c, the pump frequency is relatively blue. Therefore, the induced absorption initially is blue as well, dominating a short time over the bleaching signal. As the "holes" drift into the spectral window of the probe, the bleaching takes over, and the absorption change changes sign. The bleaching finally decays to an equilibrium value corresponding to a (locally) higher temperature of the sample.

\section{Discussion}

The observed lifetime of 1.8 ps of the OD stretch vibration of HDO: $\mathrm{D}_{2} \mathrm{O}$ is in good agreement with the value of approximately 2 ps found for the lifetime of the OD stretch in pure $\mathrm{D}_{2} \mathrm{O} .{ }^{14}$ The similarity of these numbers for the pure liquid and the isotopically diluted solution is quite surprising, since for the $\mathrm{OH}$ stretch vibration, the lifetime in pure $\mathrm{H}_{2} \mathrm{O}$ is much shorter than in the diluted solution: $\sim 200$ fs versus $740 \mathrm{fs}^{21}$ This difference is caused by the fact that in pure $\mathrm{H}_{2} \mathrm{O}$, where the distance between adjacent $\mathrm{OH}$ groups is small, the energy of an $\mathrm{OH}$ oscillator is rapidly transferred to nearby oscillators. This Förster energy transfer causes very fast spectral diffusion, and enables the vibration to find its most efficient relaxation path. Comparison of the OD stretch lifetime in $\mathrm{HDO}: \mathrm{H}_{2} \mathrm{O}$ (this study) and in pure $\mathrm{D}_{2} \mathrm{O}^{14}$ thus seems to indicate that intermolecular energy transfer plays a much smaller role for the OD stretching vibration than for the $\mathrm{OH}$ stretch. The somewhat smaller transition dipole moment of the OD stretch may at least partly explain the difference between water and deuterated water in Förster energy transfer rates.

It is an interesting observation that the lifetime of the OD stretch is more than twice as long as the $\mathrm{OH}$ stretch lifetime, since this conflicts with the energy gap law, ${ }^{22}$ which states that the vibrational lifetime is proportional to $\delta^{N}$, where $d \ll 1$ and $N$ is the number of quanta dissipated in a particular accepting mode. For a lower-frequency vibration (e.g., the OD stretch compared to the $\mathrm{OH}$ stretch), $\mathrm{N}$ will in general be smaller, implying a shorter lifetime. In this case, however, the lowerfrequency vibration lives longer than the higher-frequency vibration. It should be noted that, in addition to energy gap considerations, there are several other effects that determine the isotope effect in the vibrational lifetime, such as coupling strengths and the density-of-states of combined accepting modes.

In most vibrational relaxation processes, the energy is not transferred to a single accepting mode, but rather to a combination of modes, that may consist of one high-frequency mode that accepts the major part of the energy (such as the bending mode, $\sim 1450 \mathrm{~cm}^{-1}$ ) and several low-frequency modes to 
compensate the energy difference, or many low-frequency modes (e.g., the hydrogen-bond mode $\sim 200 \mathrm{~cm}^{-1}$ ). For highfrequency vibrations in the condensed phase, there are many combinations of accepting modes that match the energy to be dissipated and that are anharmonically coupled to the excited vibration. Hence, the relaxation rate will increase when the density of states of these combinations at the excitation energy increases.

For zeolites, ${ }^{23}$ it was found that an OD stretch mode relaxes about three time as fast as an $\mathrm{OH}$ mode. From the temperature dependence of the vibrational lifetime, the numbers of quanta of accepting modes were determined to be 5 and 3, respectively. The energy gap law would predict a much larger difference in lifetime than the observed factor of 3 . This relatively small difference could be explained from the difference in the number of accepting mode combinations, which was shown to be larger for the $\mathrm{OH}$ stretch vibration.

The isotope effect in the lifetime of the OH/OD stretching vibration can be understood by a similar argument: for isotopically diluted liquid water, apparently, the number of accepting modes is sufficiently larger for the $\mathrm{OH}$ stretching mode in HDO: $\mathrm{D}_{2} \mathrm{O}$ than for the OD stretch of $\mathrm{HDO}: \mathrm{H}_{2} \mathrm{O}$, that the $\mathrm{OH}$ stretch lifetime becomes shorter than the OD stretch lifetime, despite the larger energy gap.

It is not yet clear which are the accepting modes in the relaxation of an excited $\mathrm{OD}$ or $\mathrm{OH}$ stretching vibration. Two likely accepting modes are the HOD bending vibration, and the hydrogen-bond mode. Nienhuys et al. ${ }^{12}$ found that the temperature and frequency dependence can be well explained if the relaxation involves energy transfer to the hydrogen bond. Deàk et al. found that the energy relaxation of both the OH and OD stretch modes leads to a transient excitation of the bending mode. Unfortunately, in the present experiments, we are not able to determine the precise relaxation mechanism. Probably, both the HOD bending mode and the hydrogen-bond mode play a role in the relaxation scheme.

For liquid water, values for the spectral diffusion correlation time have been obtained of $500 \mathrm{fs}^{16}$ and $700 \mathrm{fs}^{11}$ In these studies, the $\mathrm{OH}$ stretching vibration was studied in a dilute solution of $\mathrm{HDO}$ in $\mathrm{D}_{2} \mathrm{O}$. As the frequency of the $\mathrm{OH}$ vibration is determined by the length of the hydrogen bond that binds the hydrogen atom to the oxygen atom of a nearby water molecule, ${ }^{18}$ its dynamics result from the motion of the bulk $\mathrm{D}_{2} \mathrm{O}$ molecules. In the present experiment, we studied the OD stretching vibration in a dilute solution of HDO in $\mathrm{H}_{2} \mathrm{O}$. The value for the spectral diffusion time of 400 fs directly reflects the motion of $\mathrm{H}_{2} \mathrm{O}$ molecules. In measuring this value, the OD excitation serves only as a label to follow the spectral dynamics. The fact that the vibrational lifetime is a few times as large as the spectral diffusion correlation time, allows for a very precise determination of the latter value. The shorter spectral diffusion time implies that $\mathrm{H}_{2} \mathrm{O}$ molecules show significantly faster hydrogen-bond dynamics than $\mathrm{D}_{2} \mathrm{O}$.

\section{Conclusions}

We performed two-color mid-infrared pump-probe experiments on the OD stretching vibration in HDO: $\mathrm{H}_{2} \mathrm{O}$. The data could be very well modeled using the Brownian oscillator model. From this model, the vibrational lifetime and the spectral diffusion time have been determined; their values are $1.8 \mathrm{ps}$ and $400 \mathrm{fs}$, respectively. The vibrational lifetime of the OD stretching vibration is found to be significantly longer than that of the $\mathrm{OH}$ stretching vibration. The spectral diffusion correlation time is shorter than previously obtained for $\mathrm{D}_{2} \mathrm{O}$, which means that the hydrogen-bond dynamics is faster in $\mathrm{H}_{2} \mathrm{O}$ than in $\mathrm{D}_{2} \mathrm{O}$.

Acknowledgment. This work is part of the research program of the Stichting Fundamenteel Onderzoek der Materie (Foundation for Fundamental Research on Matter) with financial support from the Nederlandse Organisatie voor Wetenschappelijk Onderzoek (Netherlands Organization for the Advancement of Scientific Research).

\section{References and Notes}

(1) Wyss, H. R.; Falk, M. Can. J. Chem. 1970, 48, 607.

(2) Ford, T. A.; Falk, M. Can. J. Chem. 1968, 46, 3579.

(3) Herzberg, G. Molecular spectra and molecular structure; D. van Nostrand: New York, 1950.

(4) Boyd, T. A. Nonlinear Optics; Academic Press: London, 1992. 87.

(5) Laubereau, A.; Greiter, L.; Kaiser, W. Appl. Phys. Lett. 1974, 25 , 385.

(6) Knudtson, J. T.; Stephenson, J. C. Chem. Phys. Lett. 1984, 107,

(7) Bakker, H. J.; Planken, P. C. M.; Kuipers, L.; Lagendijk, A. J. Chem. Phys. 1991, 94, 1736. 3413.

(8) Graener, H.; Ye, T. Q.; Laubereau, A. J. Chem. Phys. 1988, 90, 658.

(9) Woutersen, S.; Emmerichs, U.; Bakker, H. J. Science 1997, 278 ,

(10) Laenen, R.; Rauscher, C.; Laubereau, A. Phys. Rev. Lett. 1998, $80,2622$.

(11) Gale, G. M.; Gallot, G.; Hache, F.; Lascoux, N.; Bratos, S.; Leicknam, J.-C. Phys. Rev. Lett. 1999, 82, 1068.

(12) Nienhuys, H. K.; Woutersen, S.; van Santen, R. A.; Bakker, H. J. J. Chem. Phys. 1999, 111 (4), 1494

(13) Woutersen, S.; Bakker, H. J. Nature 2000, 402, 507.

(14) Deàk, J. C.; Rhea, S. T.; Iwaki, L. K.; Dlott, D. D. J. Phys. Chem. A 2000, 104, 4866 .

(15) Woutersen, S.; Emmerichs, U.; Bakker, H. J. J. Chem. Phys. 1997, 107,1483 .

(16) Woutersen, S.; Bakker, H. J. Phys. Rev. Lett. 1999, 83, 2077.

(17) Franks, F. Water; Royal Society of Chemistry: London, 1983.

(18) Mikenda, W. J. Mol. Struct. 1986, 147, 1.

(19) Mukamel, S. Nonlinear optical spectroscopy; Oxford University Press: New York, 1995.

(20) Yan, Y. J.; Mukamel, S. Phys. Rev. A 1990, 41, 6485.

(21) Lock, A. J.; Woutersen, S.; Bakker, H. J. J. Phys. Chem. A 2001, 105,1238

(22) Nitzan, A.; Mukamel, S.; Jortner, J. J. Chem. Phys. 1975, 63, 200.

(23) Brugmans, M. J. P.; Bonn, M.; Bakker, H. J.; Lagendijk, A. Chem. Phys. 1995, 201, 215. 